\title{
Towards spectral-domain optical coherence tomography on a silicon chip
}

\author{
B. I. Akca, K. Wörhoff, V. D. Nguyen, J. Kalkman, T. G. van Leeuwen, R. M. de Ridder, and M. Pollnau \\ Integrated Optical MicroSystems (IOMS) Group, MESA+ Institute for Nanotechnology, \\ University of Twente, 7500 AE Enschede, The Netherlands \\ phone: +31-53-489 2816, fax: +31-53-489 3996 \\ e-mail: b.i.akca@ewi.utwente.nl
}

Optical coherence tomography (OCT) is a widely used optical imaging technology, particularly in the medical field, since it can provide non-invasive, sub-micrometer resolution diagnostic images of tissue. Current OCT systems contain optical fibers and free-space optical components which make these instruments bulky and costly. A significant decrease in the size and cost of an OCT system is possible through the use of integrated optics, allowing for compact and low-cost OCT systems, especially suited for applications in which instrument size may play an important role.

In this work, we present a miniaturized spectral-domain OCT (SD-OCT) system. We design an arrayed waveguide grating (AWG) spectrometer in silicon oxynitride for the 1300-nm spectral range [1]. The spectral range of the SD-OCT system near $1300 \mathrm{~nm}$ is specifically selected for skin imaging. We aim at 18- $\mu \mathrm{m}$ depth resolution (determined by the full width at half maximum values of the transmission spectrum of the AWG) and a 1-mm depth range (determined by the wavelength spacing per output waveguide). The free spectral range of $78 \mathrm{~nm}$ and wavelength resolution of $0.4 \mathrm{~nm}$ of the AWG are determined to meet these requirements [2].

The fiber-based SD-OCT system with AWG spectrometer is shown in Fig. 1 [3]. The Michelson interferometer is illuminated using a superluminescent diode which has a Gaussian-like spectrum with a bandwidth of $40 \mathrm{~nm}$ and a central wavelength of $1300 \mathrm{~nm}$. Via a circulator the light is coupled into a 90/10 beamsplitter. Polarization controllers are placed into both, sample and reference arm. The backreflected light is redirected through the optical circulator to the AWG spectrometer. The collimated beam is imaged with a camera lens onto a $46 \mathrm{kHz}$ CCD linescan camera. The acquired spectra are processed by first subtracting the reference arm spectrum, then compensating the dispersion, and finally resampling to $\mathrm{k}$-space.

We achieve a depth range of $1 \mathrm{~mm}$, as shown in Fig. 2a. The measured signal-to-noise ratio (SNR) is $75 \mathrm{~dB}$. Figure $2 b$ shows the axial resolution (FWHM) determined from a Gaussian fit to the point spread function in amplitude at various depths. A slight decrease in depth resolution is observed at higher depth ranges, which we attribute to misalignment and lens aberrations. As a demonstration of OCT imaging using the AWG spectrometer, an image of a layered phantom is shown in Fig. 3. The phantom consists of three layers of scattering medium $\left(\mu_{\mathrm{s}}=4 \mathrm{~mm}^{-1}\right.$, refractive index $\left.\mathrm{n}=1.41\right)$ interleaved with non-scattering tape. We can observe all three scattering layers up to the maximum imaging depth of $1 \mathrm{~mm}$.

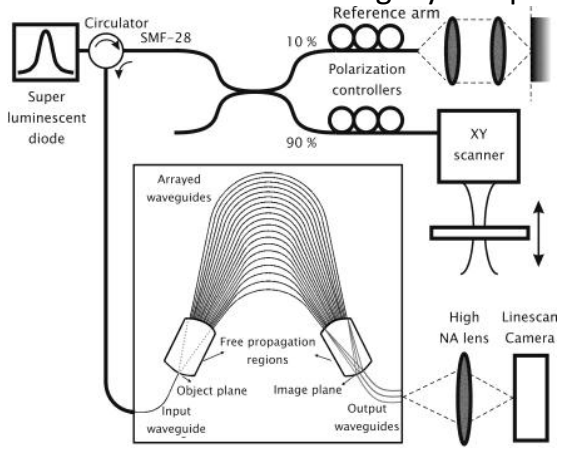

Fig. 1. Optical measurement set-up of fiberbased Michelson interferometer with integrated arrayed waveguide grating (AWG) spectrometer

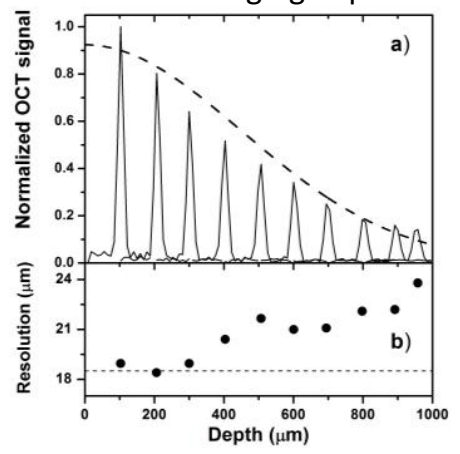

Fig. 2. In-depth (a) measured OCT signal and fit (dashed line) and (b) measured (dots) and theoretical (dashed line) axial resolution

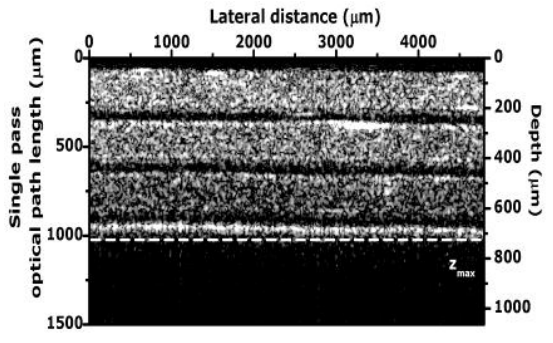

Fig. 3. An OCT image of the layered phantom measured with the AWG as spectrometer

[1] B. I. Akca, N. Ismail, G. Sengo, F. Sun, J. Kalkman, A. Driessen, M. Pollnau, K. Wörhoff, and R. M. de Ridder, "High-resolution silicon-oxynitride arrayed waveguide grating spectrometers operating around $800 \mathrm{~nm}$ and $1300 \mathrm{~nm}^{\prime \prime}$, submitted.

[2] B. I. Akca, V. D. Nguyen, J. Kalkman, T. G. van Leeuwen, K. Wörhoff, R. M. de Ridder, and M. Pollnau, "Arrayed-waveguide-grating spectrometer for spectral-domain low-coherence reflectometry", submitted.

[3] V. D. Nguyen, B. I. Akca, K. Wörhoff, R. M. de Ridder, M. Pollnau, T. G. van Leeuwen, and J. Kalkman, Opt. Lett., accepted (2011). 